\title{
A Monte-Carlo Implementation of the SAGE Algorithm for Joint Soft Multiuser and Channel Parameter Estimation
}

\author{
E. Panayirci *, A. Kocian ${ }^{\dagger}$, H. V. Poor *, and M. Ruggieri ${ }^{\dagger}$ \\ * Department of Electrical Engineering, Princeton University, Princeton, NJ 08544, USA \\ $\dagger$ University of Rome "Tor Vergata", Center for TeleInFrastructure (CTiF)-Italy, Rome, Italy
}

\begin{abstract}
An efficient, joint transmission delay and channel parameter estimation algorithm is proposed for uplink asynchronous direct-sequence code-division multiple access (DS-CDMA) systems based on the space-alternating generalized expectation maximization (SAGE) framework. The marginal likelihood of the unknown parameters, averaged over the data sequence, as well as the expectation and maximization steps of the SAGE algorithm are derived analytically. To implement the proposed algorithm, a Markov Chain Monte Carlo (MCMC) technique, called Gibbs sampling, is employed to compute the a posteriori probabilities of data symbols in a computationally efficient way. Computer simulations show that the proposed algorithm has excellent estimation performance. This so-called MCMC-SAGE receiver is guaranteed to converge in likelihood.
\end{abstract}

Index Terms- Asynchronous DS-CDMA, space-alternating generalized expectation maximization(SAGE), Markov Chain Monte Carlo (MCMC), Gibbs sampling.

\section{INTRODUCTION}

The performance of direct-sequence code-division multiple-access (DS-CDMA) transmission over mobile fading channels depends strongly on the reliability of channel parameter and quality of synchronization for each user: state-of-the-art detection algorithms that exploit multiple-access-interference and inter-symbol-interference require very powerful estimation algorithms.

Substantial amount of relevant references appeared in the literature on delay estimation. Namely, a new prospective is presented in [1] for the maximum likelihood (ML) time-delay estimation. Code timing estimation in a near-far environment for DS-CDMA systems was introduced in [2]. Joint symbol detection, time-delay and channel parameter estimation problems for asynchronous DS-CDMA systems have been investigated in several previous works (e.g., [3], [4]). Most of these works either work on one signal at a time and treat the other signals as interference, or employ a training sequence to obtain a coarse estimate of the channel parameters which is consequently used to detect data. It is clear that these approaches have disadvantages of having higher overhead and additional noise enhancement.

Some other proposed approaches for joint blind multiuser detection and channel estimation for DS-CDMA systems are subspace-based and linear prediction-based methods. Subspace-based method usually require singular value decomposition or eigenvalue decomposition which is computationally costly and does not tolerate mismatched channel parameters. Another drawback of this approach is that accurate rank determination may be difficult in a noisy environment [5], [6]. Moreover, it is not clear how these methods can be extended to include the estimation of the transmission delays jointly with the channel parameters.

E. Panayirci is on sabbatical leave from Kadir Has University, Istanbul Turkey. This research has been conducted in part within the NEWCOM++ Network of Excellence in Wireless Communications project funded through the EC 7th Framework Program, and in part under the support of the U.S. National Science Foundation under Grant CNS-06-25637.
The expectation maximization (EM) and space alternating EM (SAGE) algorithms are ideally suited to these kind of problems as they are guaranteed to converge in likelihood. Earlier work related with delay estimation based on the EM algorithm has appeared [7], [8]. Efficient iterative receiver structures are presented in [9], [10], performing joint multiuser detection and channel estimation for synchronous as well as asynchronous coded DS-CDMA systems operating over quasi-static flat Rayleigh fading channels, under the assumption that the transmissions delays are known. The Bayesian EM/SAGE algorithm can be used for joint soft-data estimation and channel estimation but the computational complexity of the resulting receiver architecture is non-polynomial in the number of users [11]. To overcome this draw-back, Hu et al. applied the Variational Bayesian EM/SAGE algorithms to joint estimation of the distributions for channel coefficients, noise variance, and information symbols for synchronous DS-CDMA in [12]. Our work may be considered to be a twofold extension of the work by Gallo et al. in [11]: First, the proposed receiver performs joint channel coefficient and transmission delay estimation within the SAGE framework. Secondly, the implication of the Monte-Carlo method in the SAGE framework makes it possible to compute soft-data estimates for all users at polynomial computational complexity, as well. Here, an efficient Markov chain Monte Carlo (MCMC) technique [13] called Gibbs sampling is used to compute the a posteriori probabilities (APP) of data symbols [14]. The APP's can be computed exactly with the MCMC algorithm, which is significantly less complex than a standard hidden Markov model approach. The resulting receiver architecture works in principal fully blind and is guaranteed to converge. For uncoded transmission, a few pilot bits must be inserted, though, to resolve the phase ambiguity problem.

The theoretical framework for the joint transmission delays and channel estimation as well as the data detection algorithms can easily be extended to coded transmission.

\section{System DESCRIPTION}

We consider an asynchronous single-rate DS-CDMA system with $K$ active users using binary phase shift keying (BPSK) modulation sharing the same propagation channel. The signal transmitted by each user experiences flat Rayleigh fading, which is assumed to be constant over the observation frame of $L$ data symbols. Each user employs a random signature waveform for transmitting symbols of duration $T_{b}$, such that each symbol consists of $N_{c}$ chips with duration $T_{c}=$ $T_{b} / N_{c}$ where $N_{c}$ is an integer. The received signal is the noisy sum of all user's contribution, delayed by the propagation delays $\tau_{k} \in$ $\left[0, T_{b} / 2\right)$, where the subscript $k$ denotes the label of the $k$ th user. After down-converting the received signal to baseband and passing it through an integrate-and-dump filter with integration time $T_{s}=$ $T_{c} / Q, Q \in \mathbb{Q}^{+}, Q N_{c}(L+1)$ samples over an observation frame of $L$ symbols are stacked into a signal column vector $\boldsymbol{r} \in \mathbb{C}^{Q N_{c}(L+1)-1}$. Note that sampling is chip-synchronous without knowledge of the 
individual transmission delays. It can therefore be expressed as

$$
r=S(\tau) \operatorname{Ad}+w .
$$

In this expression the matrix $\boldsymbol{S}(\boldsymbol{\tau}) \in \mathbb{C}^{Q N_{c}(L+1)-1 \times L K}$ contains the signature sequences of all the users

$$
\boldsymbol{S}(\boldsymbol{\tau})=\left[\begin{array}{llll}
\boldsymbol{S}_{1}\left(\tau_{1}\right), & \boldsymbol{S}_{2}\left(\tau_{2}\right), & \cdots & , \boldsymbol{S}_{K}\left(\tau_{K}\right)
\end{array}\right]
$$

where $\boldsymbol{S}_{k}\left(\tau_{k}\right) \in \mathbb{C}^{Q N_{c}(L+1)-1 \times L}$ has the form

$$
\boldsymbol{S}_{k}\left(\tau_{k}\right)=\left[\begin{array}{cccc}
\mid & \mid & & \mid \\
\boldsymbol{S}_{k}\left(\tau_{k}, 0\right) & \boldsymbol{S}_{k}\left(\tau_{k}, 1\right) & \cdots & \boldsymbol{S}_{k}\left(\tau_{k}, L-1\right) \\
\mid & \mid & & \mid
\end{array}\right]
$$

and the spreading code vector $\boldsymbol{S}_{k}\left(\tau_{k}, \ell\right) \in \mathbb{C}^{Q N_{c}(L+1)-1 \times 1}$ is given by

$$
\boldsymbol{S}_{k}\left(\tau_{k}, \ell\right)=\left[\begin{array}{c}
\mathbf{0}_{Q N_{c} \ell+\tau_{k} \times 1} \\
\mid \\
\boldsymbol{s}_{k}\left(\tau_{k}, \ell\right) \\
\mid \\
\mathbf{0}
\end{array}\right] .
$$

The vector $\boldsymbol{s}_{k}\left(\tau_{k}, \ell\right)$ contains the spreading code of user $k$ having support $\left[\ell N_{c} T_{c},(\ell+1) N_{c} T_{c}\right]$ with energy $\boldsymbol{s}_{k}^{\dagger}\left(\tau_{k}, \ell\right) \boldsymbol{s}_{k}\left(\tau_{k}, \ell\right)=1$. Finally, $\mathbf{0}_{M \times 1}$ denotes the $M \times 1$-dim. all-zero column vector.

The block diagonal channel matrix $\boldsymbol{A} \in \mathbb{C}^{L K \times L K}$ in (1) is given by $\boldsymbol{A}=\operatorname{diag}\left\{\boldsymbol{A}_{1}, \cdots, \boldsymbol{A}_{K}\right\}$. The channel matrix for user $k$, $\boldsymbol{A}_{k} \in \mathbb{C}^{L \times L}$, is given by $\boldsymbol{A}_{k}=\mathbf{I}_{L} \otimes a_{k}$ where $\mathbf{I}_{\mathbf{L}}$ is the $L$-dim. identity matrix, and the symbol $\otimes$ denotes the Kronecker product. The $k$ th user's channel coefficient $a_{k}$ is a circularly symmetric complex Gaussian random variable with zero mean and variance $\sigma_{k}^{2}$. The $k$ th user's transmission delay is assumed to be uniformly distributed.

The symbol vector $\boldsymbol{d} \in \mathbb{C}^{L K}$ takes the form $\boldsymbol{d}=\operatorname{col}\left\{\boldsymbol{d}_{1}, \cdots, \boldsymbol{d}_{K}\right\}$ where the vector $\boldsymbol{d}_{k} \in \mathbb{C}^{L}$ contains the $k$ th user's symbols, i.e. $\boldsymbol{d}_{k}=$ $\operatorname{col}\left\{d_{k}(0), \cdots, d_{k}(L-1)\right\}$ with $d_{k}(\ell) \in\{-1,+1\}$ denoting the symbol transmitted by the $k$ th user during the $\ell$ th signalling interval. Finally, the column vector $\boldsymbol{w} \in \mathbb{C}^{Q N_{c}(L+1)-1}$ contains complex, circularly symmetric white Gaussian noise having covariance matrix $N_{0} \mathbf{I}$. We assume that the vectors $\boldsymbol{a} \triangleq \operatorname{col}\left\{a_{1}, a_{2}, \cdots, a_{K}\right\}, \boldsymbol{\tau} \triangleq$ $\operatorname{col}\left\{\tau_{1}, \tau_{2}, \cdots, \tau_{K}\right\}, \boldsymbol{d}$ and $\boldsymbol{w}$ and their components are independent. The receiver does not know the data sequences, the (complex) channel coefficients, or the transmission delays.

\section{Monte-Carlo SAGE Joint Parameter Estimation}

\section{A. The SAGE Algorithm}

In previous applications, the SAGE algorithm [15] has been extensively used to iteratively approximate the ML/MAP estimate of a parameter vector $\boldsymbol{\theta}$ with respect to the observed data $\boldsymbol{r}$. To obtain a receiver architecture that iterates between soft-data and channel estimation, one might choose the parameter vector as $\boldsymbol{\theta}=$ $\left\{\mathfrak{R}\left(a_{1}\right), \cdots, \mathfrak{R}\left(a_{K}\right), \mathfrak{I}\left(a_{1}\right), \cdots, \mathfrak{I}\left(a_{K}\right), \tau_{1}, \cdots, \tau_{K}\right\}$. The symbols $\mathfrak{R}(\cdot)$ and $\mathfrak{I}(\cdot)$ denote the real and imaginary parts of the complex argument, respectively. At iteration $i$, only the parameter vector of user $k, \boldsymbol{\theta}_{k}$ are updated, while the parameter vectors of the other users $\boldsymbol{\theta}_{\bar{k}}=\boldsymbol{\theta} \backslash \boldsymbol{\theta}_{k}$ are kept fixed. In the SAGE framework $\boldsymbol{r}$ is referred to as the incomplete data. The so-called admissible hidden data $\chi_{k}$ with respect to $\boldsymbol{\theta}$ is selected to be $\chi_{k}=\{\boldsymbol{r}, \boldsymbol{d}\}$. Notice that $\chi_{k}$ can only be partially observed. Applying the SAGE algorithm to MAP parameter estimation, yields the expectation (E)-step

$$
Q_{k}\left(\boldsymbol{\theta}_{k}, \boldsymbol{\theta}^{[i]}\right)=E_{\boldsymbol{d}}\left\{\log p\left(\boldsymbol{r}, \boldsymbol{d}, \boldsymbol{a}_{k}, \boldsymbol{\tau}_{k}, \boldsymbol{a}_{\bar{k}}^{[i]}, \boldsymbol{\tau}_{\bar{k}}^{[i]}\right) \mid \boldsymbol{r}, \boldsymbol{a}^{[i]}, \boldsymbol{\tau}^{[i]}\right\} .
$$

The maximization (M)-step computes a value of the argument $\tau_{k}$ in (2) to obtain the update $\boldsymbol{\theta}_{k}^{[i+1]}$. The objective function is nondecreasing at each iteration.

\section{B. The Monte-Carlo SAGE algorithm}

We will see that direct computation of the expectation in 2 requires a non-polynomial number of operations in the number of users $K$ and thus becomes prohibitive with increasing $K$. To make the computation of the expectation in 21 feasible though, we propose to use the technique of Markov chain Monte Carlo (MCMC) to obtain the Monte-Carlo SAGE algorithm. MCMC is a statistical technique that allows generation of ergodic pseudo-random samples $\boldsymbol{d}^{[i, 1]}, \ldots, \boldsymbol{d}^{\left[i, N_{t}\right]}$ from the current approximation to the conditional pdf $p\left(\boldsymbol{d} \mid \boldsymbol{r}, \boldsymbol{\theta}^{[i]}\right)$. These samples are used to approximate the expectation in (2) by the sample-mean. The Gibbs sampler and the Metropolis-Hastings algorithm are widely used MCMC algorithms. Here we describe only the Gibbs sampler [16], [14], as it is the most commonly used in applications. Having initialized $\boldsymbol{d}^{[0,0]}$ randomly, the Gibbs sampler iterates the following loop at SAGE iteration $i$ :

- Draw sample $\boldsymbol{d}_{1}^{[i, t]}$ from $p\left(\boldsymbol{d}_{1} \mid \boldsymbol{d}_{2}^{[i, t-1]}, \ldots, \boldsymbol{d}_{K}^{[i, t-1]}, \boldsymbol{r}, \boldsymbol{\theta}^{[i]}\right)$

- Draw sample $\boldsymbol{d}_{2}^{[i, t]}$ from $p\left(\boldsymbol{d}_{2} \mid \boldsymbol{d}_{1}^{[i, t]}, \boldsymbol{d}_{3}^{[i, t-1]} \ldots, \boldsymbol{d}_{K}^{[i, t-1]}, \boldsymbol{r}, \boldsymbol{\theta}^{[i]}\right)$

- Draw sample $\boldsymbol{d}_{K}^{[i, t]}$ from $p\left(\boldsymbol{d}_{K} \mid \boldsymbol{d}_{1}^{[i, t]}, \ldots, \boldsymbol{d}_{K-1}^{[i, t]}, \boldsymbol{r}, \boldsymbol{\theta}^{[i]}\right)$

Following this approach, we have

$$
Q_{k}\left(\boldsymbol{\theta}_{k}, \boldsymbol{\theta}^{[i]}\right)=\frac{1}{N_{t}} \sum_{t=1}^{N_{t}}\left\{\log p\left(\boldsymbol{r}, \boldsymbol{d}^{[i, t]}, \boldsymbol{a}_{k}, \boldsymbol{\tau}_{k}, \boldsymbol{a}_{\bar{k}}^{[i]}, \boldsymbol{\tau}_{\bar{k}}^{[i]}\right)\right\} .
$$

Notice that with increasing $N_{t}$, the Monte-Carlo SAGE algorithm converges to the MAP solution $\boldsymbol{\theta}=\boldsymbol{\theta}^{\star}$ up to random fluctuations around $\boldsymbol{\theta}^{\star}$ [17].

\section{Receiver design}

This subsection is devoted to the derivation of a receiver architecture for joint estimation of parameters within the Monte-Carlo SAGE framework. Discarding terms independent of $\boldsymbol{a}$ and $\tau$, we obtain

$\log p(\boldsymbol{r}, \boldsymbol{d}, \boldsymbol{a}, \boldsymbol{\tau})=\log p(\boldsymbol{r} \mid \boldsymbol{d}, \boldsymbol{a}, \boldsymbol{\tau})+\log p(\boldsymbol{d})+\log p(\boldsymbol{a})+\log p(\boldsymbol{\tau})$.

From [1, it follows that

$$
\log p(\boldsymbol{r} \mid \boldsymbol{a}, \boldsymbol{\tau}, \boldsymbol{d}) \propto \Re\left\{\boldsymbol{r}^{\dagger} \boldsymbol{S} \boldsymbol{A} \boldsymbol{d}\right\}-\frac{1}{2} \boldsymbol{\mu}(\boldsymbol{\theta}, \boldsymbol{d})^{\dagger} \boldsymbol{\mu}(\boldsymbol{\theta}, \boldsymbol{d}),
$$

where $\boldsymbol{\mu}(\boldsymbol{\theta}, \boldsymbol{d}) \triangleq \sum_{k=1}^{K} \sum_{\ell=0}^{L-1} \boldsymbol{S}_{k}\left(\ell, \tau_{k}\right) a_{k} d_{k}(\ell)$ and $(.)^{\dagger}$ is the conjugate transpose of the argument.

1) The E-step: Substituting (4) into 37 yields after some algebraic manipulations for the E-step of the Monte-Carlo SAGE algorithm:

$$
\begin{aligned}
& Q_{k}\left(\boldsymbol{\theta}_{k} \mid \boldsymbol{\theta}^{[i]}\right)= \\
& \frac{2}{N_{0}} \sum_{\ell=0}^{L-1} \Re\left\{a_{k}^{*} \Psi\left(\ell, \tau_{k}\right)\right\}-\frac{L}{N_{0}}\left|a_{k}\right|^{2}-\frac{1}{\sigma_{k}^{2}}\left|a_{k}\right|^{2}
\end{aligned}
$$

with the branch definition

$$
\Psi\left(\ell, \tau_{k}\right) \triangleq \boldsymbol{S}_{k}^{\dagger}\left(\ell, \tau_{k}\right)\left(\tilde{d}_{k}^{[i]}(\ell) \boldsymbol{r}-\mathcal{I}_{k}^{[i]}(\ell)\right)
$$


and the interference term

$$
\begin{aligned}
\mathcal{I}_{k}^{[i]}(\ell) \triangleq \sum_{k^{\prime} \neq k} a_{k}^{\prime[i]}\left(\boldsymbol{S}_{k^{\prime}}\left(\ell+1, \tau_{k^{\prime}}^{[i]}\right)\left(d_{k}(\ell) \widetilde{d_{k^{\prime}}(\ell}+1\right)\right)^{[i]} \\
+\boldsymbol{S}_{k^{\prime}}\left(\ell, \tau_{k^{\prime}}^{[i]}\right)\left(\widehat{\left.d_{k}(\ell) d_{k^{\prime}}(\ell)\right)^{[i]}}\right. \\
\left.\left.+\boldsymbol{S}_{k^{\prime}}\left(\ell-1, \tau_{k^{\prime}}^{[i]}\right)\left(d_{k}(\ell) \widehat{d_{k^{\prime}}(\ell}-1\right)\right)^{[i]}\right) .
\end{aligned}
$$

Moreover,

$$
\tilde{d}_{k}^{[i]}(\ell) \triangleq \sum_{m \in \mathcal{S}} m P\left(d_{k}(\ell)=m \mid \boldsymbol{r}, \boldsymbol{\tau}^{[i]}, \boldsymbol{a}^{[i]}\right)
$$

and

$$
\begin{aligned}
& \left(d_{k} \widetilde{(\ell) d_{k^{\prime}}}\left(\ell^{\prime}\right)\right)^{[i]} \triangleq \sum_{m \in \mathcal{S}} \sum_{n \in \mathcal{S}} m n \\
& \quad \times P\left(d_{k}(\ell)=m, d_{k^{\prime}}\left(\ell^{\prime}\right)=n \mid \boldsymbol{r}, \boldsymbol{\tau}^{[i]}, \boldsymbol{a}^{[i]}\right), \text { for } k^{\prime} \neq k,
\end{aligned}
$$

where $\mathcal{S} \triangleq\{-1,+1\}$ is the signal constellation and the lag is within range $\ell^{\prime} \in\{\ell-1, \ell, \ell+1\}$.

2) The M-step: The M-step of the SAGE algorithm is realized by first maximizing (5) with respect to the transmission delays $\tau_{k}$,

$$
\tau_{k}^{(i+1)}=\arg \max _{\tau_{k}}\left|\sum_{\ell=0}^{L-1} \Psi\left(\ell, \tau_{k}\right)\right| .
$$

Then by inserting (8) into [5], taking derivatives with respect to the $a_{k}$ 's, setting the results equal to zero, and solving yields

$$
a_{k}^{(i+1)}=\frac{1}{L+N_{0} / \sigma_{k}^{2}} \sum_{\ell=0}^{L-1} \Psi\left(\ell, \tau_{k}^{(i+1)}\right) .
$$

\section{Monte-Carlo Implementation to the Computation of} A Posteriori Probabilities

\section{A. Computation of the soft-data symbols in (6)}

Let $\overline{\boldsymbol{d}_{k}(\ell)} \triangleq \boldsymbol{d} \backslash\left\{d_{k}(\ell)\right\}$. For notational simplicity we use $\overline{\boldsymbol{d}} \triangleq$ $\overline{d_{k}(\ell)}$ throughout this section. Then, the a posteriori probability of $d_{k}(\ell)$ in 6 can be evaluated as

$$
\begin{aligned}
& P\left(d_{k}(\ell)=m \mid \boldsymbol{r}, \boldsymbol{\tau}^{[i]}, \boldsymbol{a}^{[i]}\right) \\
& =\sum_{\overline{\boldsymbol{d}}} P\left(d_{k}(\ell)=m \mid \overline{\boldsymbol{d}}, \boldsymbol{r}, \boldsymbol{\tau}^{[i]}, \boldsymbol{a}^{[i]}\right) P\left(\overline{\boldsymbol{d}} \mid \boldsymbol{r}, \boldsymbol{\tau}^{[i]}, \boldsymbol{a}^{[i]}\right) \\
& \approx \frac{1}{N_{t}} \sum_{t=1}^{N_{t}} P\left(d_{k}(\ell)=m \mid \overline{\boldsymbol{d}}^{[i, t]}, \boldsymbol{r}, \boldsymbol{\tau}^{[i]}, \boldsymbol{a}^{[i]}\right) .
\end{aligned}
$$

To compute $P\left(d_{k}(\ell)=m \mid \overline{\boldsymbol{d}}^{[i, t]}, \boldsymbol{r}, \boldsymbol{\tau}^{[i]}, \boldsymbol{a}^{[i]}\right)$ for this Markov chain Rao-Blackwellization technique, we define

$$
\lambda^{[i, t]} \triangleq \ln \frac{P\left(d_{k}(\ell)=+1 \mid \overline{\boldsymbol{d}}^{[i, t]}, \boldsymbol{r}, \boldsymbol{\tau}^{[i]}, \boldsymbol{a}^{[i]}\right)}{P\left(d_{k}(\ell)=-1 \mid \overline{\boldsymbol{d}}^{[i, t]}, \boldsymbol{r}, \boldsymbol{\tau}^{[i]}, \boldsymbol{a}^{[i]}\right)},
$$

For uncoded transmission, the data symbols are i.i.d. and equally likely. Therefore, it follows from (10) that

$$
\lambda^{[i, t]}=\ln \frac{P\left(\boldsymbol{r} \mid d_{k}(\ell)=+1, \overline{\boldsymbol{d}}^{[i, t]}, \boldsymbol{\tau}^{[i]}, \boldsymbol{a}^{[i]}\right)}{P\left(\boldsymbol{r} \mid d_{k}(\ell)=-1, \overline{\boldsymbol{d}}^{[i, t]}, \boldsymbol{\tau}^{[i]}, \boldsymbol{a}^{[i]}\right)},
$$

from which it can be easily seen that

$$
P\left(d_{k}(\ell)=m \mid \overline{\boldsymbol{d}}^{(t)}, \boldsymbol{r}, \boldsymbol{\tau}^{[i]}, \boldsymbol{a}^{[i]}\right)=\frac{1}{1+\exp \left(-m \lambda^{[i, t]}\right)} .
$$

From (1), we have $p(\boldsymbol{r} \mid \boldsymbol{D}) \sim \exp \left(-\frac{1}{N_{0}}|\boldsymbol{r}-\boldsymbol{G} \boldsymbol{d}|^{2}\right)$, with $\boldsymbol{G} \triangleq$ $\mathrm{S}(\boldsymbol{\tau}) \boldsymbol{A}$ and $\boldsymbol{d}=\operatorname{col}\left\{\boldsymbol{d}_{1}, \boldsymbol{d}_{2}, \cdots, \boldsymbol{d}_{K}\right\}$, . After some algebra (11) can be expressed as

$$
\lambda^{[i, t]}=\frac{4}{N_{0}} \Re\left\{\left(\boldsymbol{g}_{q}^{[i]}\right)^{\dagger}\left(\boldsymbol{r}-\boldsymbol{G}_{\bar{q}}^{[i]} \boldsymbol{d}_{\bar{q}}^{[i, t]}\right)\right\},
$$

where $q \triangleq k L+\ell$, and $\boldsymbol{G}_{\bar{q}}$ is $\boldsymbol{G}$ with its $q$ th column $\boldsymbol{g}_{q}$ removed. Similarly, $\boldsymbol{d}_{\bar{q}}$ denotes the vector $\boldsymbol{d}$ with its $q$ th component removed.

In summary, for each $k=1,2, \cdots, K$ and $\ell=0,1, \cdots, L-1$, to estimate the a posteriori probabilities $P\left(d_{k}(\ell) \mid \boldsymbol{r}, \boldsymbol{\tau}^{[i]}, \boldsymbol{a}^{[i]}\right)$ in (9), the Gibbs sampler runs over all symbols $N_{t}$ times to generate a collection of vectors $\left\{\overline{\boldsymbol{d}}^{[i, t]} \triangleq \overline{\boldsymbol{d}}_{k}^{[i, t]}(\ell)\right\}_{t=1}^{N_{t}}$ which are used in 12 to estimate the desired quantities.

B. Computation of the soft-value for the product of two data symbols in (7)

Similarly, a number of random samples $\overline{\overline{\boldsymbol{d}}}^{[i, t]} \triangleq{\overline{\overline{\boldsymbol{d}_{k, k^{\prime}}\left(\ell^{\prime}\right)}}}^{i, t]}, t=$ $1,2, \cdots, N_{t}, \ell^{\prime} \in\{-1,0,+1\}$ are drawn, using the Gibbs sampling technique, from the joint conditional posterior distribution, $P(\overline{\bar{d}} \mid$ $\left.\boldsymbol{r}, \boldsymbol{\tau}^{[i]}, \boldsymbol{a}^{[i]}\right)$. Based on the samples $\overline{\overline{\boldsymbol{d}}}^{[i, t]},\left(d_{k} \widetilde{(\ell) d_{k^{\prime}}}\left(\ell^{\prime}\right)\right)^{[i]}$ in 7 can be evaluated by

$$
\begin{aligned}
& \left(d_{k} \widehat{(\ell) d_{k^{\prime}}}\left(\ell^{\prime}\right)\right)^{[i]} \approx\left(1 / N_{t}\right) \\
& \times \sum_{t=1}^{N_{t}} \sum_{m, n \in \mathcal{S}} m n P\left(d_{k}(\ell)=m, d_{k^{\prime}}\left(\ell^{\prime}\right)=n \mid \overline{\overline{\boldsymbol{d}}}^{[i, t]}, \boldsymbol{r}, \boldsymbol{\tau}^{[i]}, \boldsymbol{a}^{[i]}\right) .
\end{aligned}
$$

We need to evaluate the probability in the expression above. Following the same route taken as in the previous section and after some algebra, it can be expressed as

$$
\begin{array}{r}
P\left(d_{k}(\ell)=m, d_{k^{\prime}}\left(\ell^{\prime}\right)=n \mid \overline{\overline{\boldsymbol{d}}}^{[i, t]}, \boldsymbol{r}, \boldsymbol{\tau}^{[i]}, \boldsymbol{a}^{[i]}\right)= \\
\frac{1}{1+\exp \left(-\zeta^{[i, t]}\right)} \cdot \frac{1}{1+\exp \left(-\lambda^{[i, t]}\right)} .
\end{array}
$$

The quantities $\zeta^{[i, t]}$ and $\lambda^{[i, t]}$ 13 are given by

$$
\begin{aligned}
\zeta^{[i, t]} & =\frac{4}{N_{0}} \Re\left\{n\left(\boldsymbol{g}_{p}^{[i]}\right)^{\dagger}\left(\boldsymbol{r}-\boldsymbol{G}_{\overline{p, q}}^{[i]} \boldsymbol{d}_{\overline{p, q}}^{[i, t]}\right)-m n\left(\boldsymbol{g}_{p}^{[i]}\right)^{\dagger} \boldsymbol{g}_{q}^{[i]}\right\}, \\
\lambda^{[i, t]} & =\frac{4}{N_{0}} \Re\left\{m\left(\boldsymbol{g}_{q}^{[i]}\right)^{\dagger}\left(\boldsymbol{r}-\boldsymbol{G}_{\bar{q}}^{[i]} \boldsymbol{d}_{\bar{q}}^{[i, t]}\right)\right\},
\end{aligned}
$$

where $p \triangleq k^{\prime} L+\ell^{\prime}$ and $q \triangleq k L+\ell$. $\boldsymbol{G}_{\overline{p, q}}$ is $\boldsymbol{G}$ with its $p$ th and $q$ th columns $\boldsymbol{g}_{p}, \boldsymbol{g}_{q}$ removed. Similarly, $\boldsymbol{d}_{\overline{p, q}}$ denotes the vector $\boldsymbol{d}$ with its $p$ th and $q$ th components removed.

\section{PERformance ANALYSis}

\section{A. Modified Cramer-Rao Bounds for the Estimated Parameters}

We now derive the modified Cramer-Rao lower bounds (MCRB) on the variances of any unbiased estimates $\widehat{\boldsymbol{\theta}}$ of the parameter vector $\boldsymbol{\theta}$. It is shown in [18] that for $\theta_{p} \in \boldsymbol{\theta}, \operatorname{var}\left(\widehat{\theta}_{p}-\theta_{p}\right) \geq\left[\boldsymbol{I}^{-1}(\boldsymbol{\theta})\right]_{p p}$, where $\boldsymbol{I}(\boldsymbol{\theta})$ is the $3 K \times 3 K$ Fisher information matrix whose $(p, q)$ th component is defined by

$$
[\boldsymbol{I}(\boldsymbol{\theta})]_{p q} \triangleq-E_{\boldsymbol{r}, \boldsymbol{a}}\left\{\frac{\partial^{2} \ln p(\boldsymbol{r}, \boldsymbol{a} \mid \boldsymbol{\tau})}{\partial \theta_{p} \partial \theta_{q}}\right\}, \text { for } p, q=1,2, \cdots, 3 K .
$$

For the joint likelihood function in [4, it is shown in [19] that the Fisher information matrix can be computed by

$$
\begin{aligned}
& \quad[\boldsymbol{I}(\boldsymbol{\theta})]_{p q}=\frac{2}{N_{0}} E_{\boldsymbol{d}}\left\{E_{\boldsymbol{a} \mid \boldsymbol{d}}\left\{\Re\left[\frac{\partial \boldsymbol{\mu}^{\dagger}(\boldsymbol{\theta}, \boldsymbol{d})}{\partial \theta_{p}} \frac{\partial \boldsymbol{\mu}(\boldsymbol{\theta}, \boldsymbol{d})}{\partial \theta_{q}}\right]\right\}\right\}, \\
& p, q=1,2, \cdots, 3 K .
\end{aligned}
$$


Taking the expectations with respect to channel coefficients $\boldsymbol{a}$ and data $\boldsymbol{d}$ after taking the partial derivatives in (14) with respect to $\theta_{p}$ and $\theta_{q}$, for different regions of $p$ and $q$ values, under the assumption that the data sequences are independent and equally likely and the fact that $\boldsymbol{S}^{\dagger}\left(\tau_{p}, \ell\right) \boldsymbol{S}\left(\tau_{p}, \ell\right)=1$, for $p=1,2 \cdots K ; \ell=0,1, \cdots, L-1$, the Fisher information matrix becomes a diagonal matrix whose $(p, p)$ th component can be evaluated as

$$
[\boldsymbol{I}(\boldsymbol{\theta})]_{p p}=\frac{2}{N_{0}}\left\{\begin{array}{lrl}
L ; & p & =1, \cdots, K \\
L ; & p & =K+1, \cdots, 2 K \\
\sigma_{p}^{2} \sum_{\ell=0}^{L-1}\left|\boldsymbol{S}^{\prime}(\ell)\right|^{2} ; & p=2 K+1, \cdots, 3 K .
\end{array}\right.
$$

with the short-cut $\left.\boldsymbol{S}^{\prime}[\ell] \triangleq \frac{\partial \boldsymbol{S}_{p}\left(\tau_{p}, \ell\right)}{\partial \tau_{p}}\right|_{t=\ell T_{b}+\widehat{\tau}_{p}}$. The final result for the MCRBs on the estimates of the channel coefficients and the transmission delays is obtained by inverting the diagonal matrix $\boldsymbol{I}(\boldsymbol{\theta})$ in 15 as follows.

$$
\begin{aligned}
& \operatorname{var}\left(\widehat{a}_{k}\right) \geq N_{0} / L, \\
& \operatorname{var}\left(\widehat{\tau}_{k}\right) \geq 1 /\left(8 \pi^{2} L \overline{\gamma_{k}} B_{s_{k}}^{2}\right),
\end{aligned}
$$

$k=1,2, \ldots, K$. The symbol $\overline{\gamma_{k}} \triangleq \sigma_{k}^{2} / N_{0}$ is the average SNR, $B_{s_{k}}$ is the Gabor bandwidth of the $k$ th user's spreading code waveform, $s_{k}(t)$ i.e.,

$$
B_{s_{k}} \triangleq\left(\int_{-\infty}^{+\infty} f^{2}\left|S_{k}(f)\right|^{2} d f\right)^{1 / 2},
$$

and $S_{k}(f)$ is the Fourier transform of $s_{k}(t), t \in\left[0, T_{b}\right]$. Note that the Gabor bandwidth $B_{s_{k}}$ tends to infinity for rectangular-shaped (continuous-time) chip waveforms.

\section{B. Numerical Examples}

To assess the performance of the proposed (non-linear) MonteCarlo SAGE scheme, an asynchronous uncoded DS-CDMA system with $K=5$ users, rectangular chip waveforms with processing gain $N_{c}=8$, and $L=80$ transmitted symbols per block is considered. The receiver processes $Q=12$ samples per chip. For each data block, Gibbs sampling is performed over 50 iterations. A few, say $L_{p}=4$ pilot symbols are embedded in each block to overcome the phase ambiguity problem. Each user's strongest path from the MMSE estimate of $\boldsymbol{a}$ given the $K Q\left(L_{p}+1\right)-1$ samples of $\boldsymbol{r}$ and the pilot symbols, yield the initial estimates $\boldsymbol{a}^{[0]}$ and $\tau^{[0]}$. The MMSE estimate of $\boldsymbol{d}$, given $\boldsymbol{r}$ and weighted by $\boldsymbol{a}^{[0]}$ yields the initial symbol estimate $\boldsymbol{d}^{[0]}$. We refer to this method as MMSE-separate estimation (MMSE-SE). For comparison purpose, the SAGE-scheme for joint data detection and channel estimation in [10] for known transmission delays and hard-decision decoding has also been considered subsequently. We refer to these scheme as "SAGE-JDE, $\tau$ known".

To study the behavior of the proposed MCMC-SAGE scheme, we consider communication over AWGN (not known to the receiver). The individual powers are given by

$$
\begin{array}{ll}
\sigma_{1}^{2}=-4 \mathrm{~dB}, & \sigma_{2}^{2}=-2 \mathrm{~dB}, \quad \sigma_{3}^{2}=0 \mathrm{~dB}, \\
\sigma_{4}^{2}=+2 \mathrm{~dB}, & \sigma_{5}^{2}=+4 \mathrm{~dB},
\end{array}
$$

Fig. 1 shows the mean-square-error (MSE) of the channel estimates $\widehat{\boldsymbol{a}}_{1}$ (weakest user) and $\widehat{\boldsymbol{a}}_{3}$ (normal user) as a function of the normalized transmission delays $\tau / T_{b}$ which are uniformly distributed on the interval between zero and the value on the abscissa. It can be seen that the MCMC-SAGE performs close to the MCRB over the entire range of $\tau$. Not shown in the plot, convergence is achieved after around 25 iterations i.e., every user's parameter vector is updated five times.

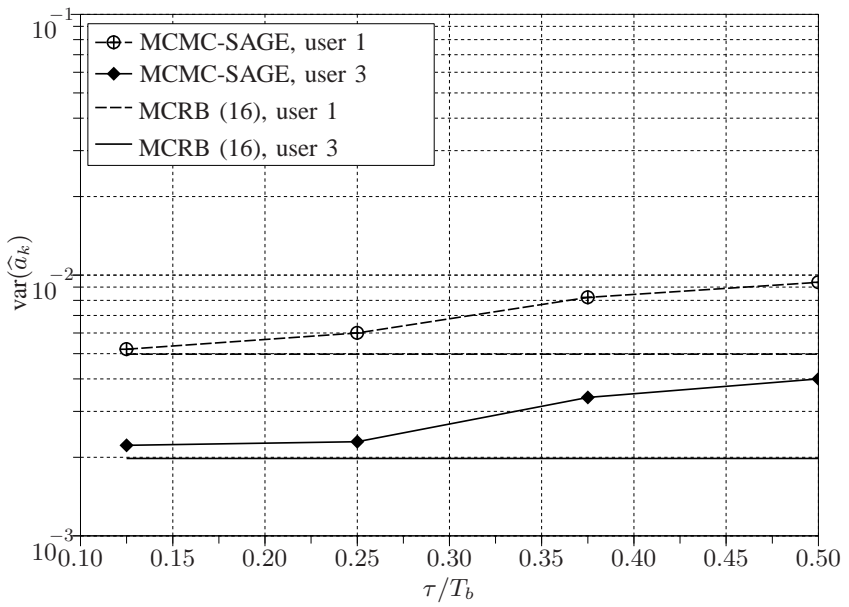

Fig. 1. $\operatorname{var}\left(\widehat{a}_{k}\right)$ of the MCMC-SAGE in near-far scenario.

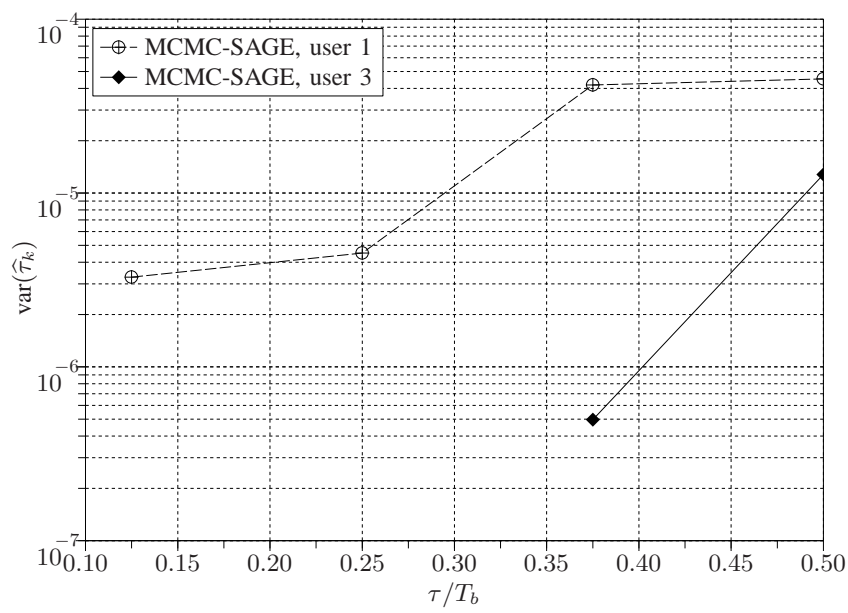

Fig. 2. $\operatorname{var}\left(\widehat{\tau}_{k}\right)$ of the MCMC-SAGE in near-far scenario.

Fig. 2 depicts the MSE of the delay estimates $\widehat{\tau}_{1}$ and $\widehat{\tau}_{3}$. Notice that the MCRB for $\tau$ tends to zero for time-continuous signature waveforms. It can be seen that user 3 does not encounter delay estimation errors for small transmission delays i.e., $\tau / T_{b} \leq 0.2$. This effect can be partially explained by the large number of samples per chip i.e., $Q=12$. Though for higher transmission delays, $\operatorname{var}\left(\widehat{\tau}_{3}\right)$ is finite, because of the increasing residual interference in the receiver.

The bit-error-rate $(\overline{\mathrm{BER}})$ of the proposed receiver is plotted in Fig. 3 versus the effective SNR $\frac{L-L_{p}}{L} \bar{\gamma}_{k}, \bar{\gamma}_{k} \triangleq \sigma_{k}^{2} / N_{0}, k=$ $1, \ldots, K$. The transmission delays are uniformly distributed on $\left[0, T_{b} / 2\right)$. It can be seen that the MMSE-SDE scheme cannot handle delay estimation errors at all due to high correlations between the users' signature sequences. The proposed MCMC-SAGE scheme and the "SAGE-JDE, $\tau$ known" perform similar. The weakest user 1 performs close to the single-user (SU) bound. The normal user 3 has a multiuser efficiency of roughly $1 \mathrm{~dB}$ over the entire range of SNR values. 

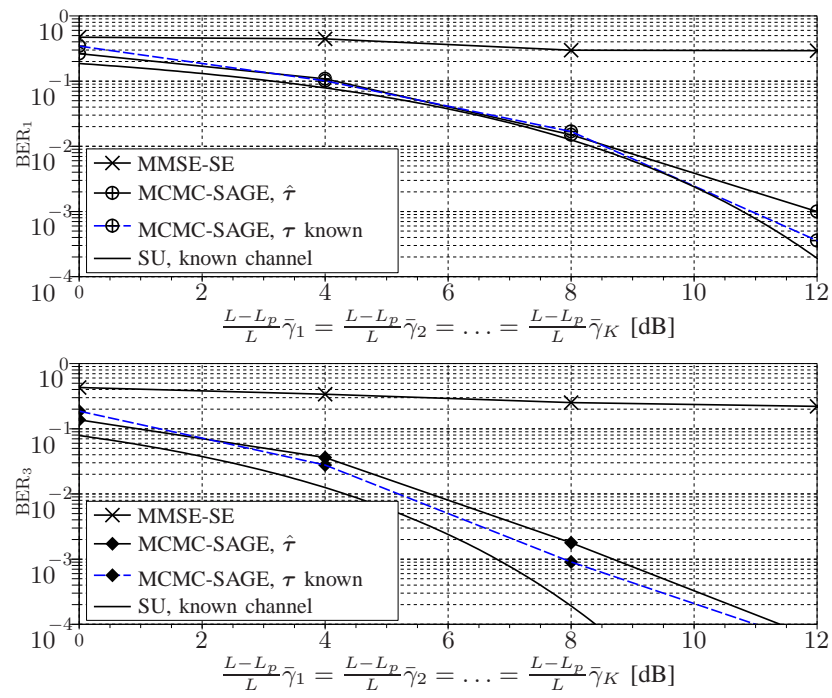

Fig. 3. BER-performance in near-far scenario.

\section{CONCLUSIONS}

A computationally efficient estimation algorithm has been proposed for estimating the transmission delays and the channel coefficients jointly in a non-data-aided fashion via the SAGE algorithm. The a posteriori probabilities needed to implement the SAGE algorithm have been computed by means of the Gibbs sampling technique. Exact analytical expression have been obtained for the estimates of transmission delays and channel coefficients. At each iteration the likelihood function is non-decreasing.

\section{REFERENCES}

[1] J. A. Stuller and N. Hubing. New perspectives for maximum likelihood time-delay estimation. IEEE Trans. Signal Processing, 45:513-525, March 1991.

[2] R. F. Smith and S. L. Miller. Code timing estimation in a near-far environment for direct-sequence code-division multiple-access. Military Communications Conf., 1994. MILCOM '94. Conference Record, 1:47 - 51, 2-5 Oct. 1994.

[3] A. Ranheim and P. Pelin. Joint symbol detection and channel parameter estimation in asynchronous DS-CDMA systems. IEEE Trans. Signal Process., 48(2):545-550, Feb. 2000.

[4] D. Zheng, J. Li and S. L. Miller. An efficient code-timing estimator for DS-CDMA signals. IEEE Trans. Signal Process., 45:82-89, Jan. 1997.

[5] S. E. Bensley et. al. Suspace-based channel estimation for code-division multiple access communications systems. IEEE Trans. Signal Process., 44:1009-1020, Aug. 1996.

[6] E. G.Strom, S. Parkvall, S. L. Miller, and B. E. Ottersten. Propagation delay estimation in asynchronous direct-sequence code-division multiple access systems. IEEE Trans. Commun., 44:84-93, Jan. 1996.

[7] C. N. Georghiades. Optimum delay and sequence estimation from incomplete data. IEEE Trans. Information Theory, 36:202-208, January 1990.

[8] C. N. Georghiades and D. L. Snyder. The expectation-maximization algorithm for symbol unsynchronized sequence detection. IEEE Trans. Communications, 39:54-61, January 1991.

[9] A. Kocian and B. H. Fleury. EM-based joint data detection and channel estimation of DS-CDMA signals. IEEE Trans. Commun., 51(10):17091720, 2003.

[10] A. Kocian, I. Land and B. H. Fleury. Joint channel estmation partial succesive interference cancellation and data decoding for DS-CDMA based on SAGE algorithm. IEEE Trans. Commun., 55(6):1231-1241, June 2004.

[11] A. S. Gallo, G. M. Vitetta, and E. Chiavaccini. A BEM-based algorithm for soft-in soft-output detection of co-channel signals. IEEE Trans. Wireless Communications, 3(5):1533-1542, 2004.
[12] B. Hu, I. Land, L. Rasmussen, R. Piton, and B.H. Fleury. A divergence minimization approach to joint multiuser decoding for coded CDMA. IEEE Journal on Selected Areas in Communications, 26(3):432-445, 2008.

[13] A. Gelfand and A. Smith. Sampling-based approaches to calculating marginal densities. J. Amer. Stat. Assoc., 85:398-409, 1990.

[14] A. Doucet and X. Wang. "Monte Carlo methods for signal processing". IEEE Signal Process. Magazine, 153:152-170, Nov. 2005.

[15] J. A. Fessler and A. O. Hero. Space-alternating generalized expectationmaximization algorithm. IEEE Trans. Signal Process., 42(10):26642677, 1994.

[16] B. F. Boroujeny, H. Zhu and Z. Shi. Iterative multiuser receivers for CDMA channels: an EM-basedapproach. IEEE Trans. Signal Process., 54(5):1896-1909, 2006.

[17] G. Wei and M. Tanner. A Monte-Carlo implementation of the EM algorithm and the poor man's data augmentation algorithms. Journal of American Stat. Assoc., 85(411):699-704, 1990.

[18] H. L. Van Trees. Detection, Estimation and Modulation Theory. Wiley, New York, 1968.

[19] S. M. Kay. Fundamentals of Statsitical Signal Processing: Estimation Theory. Prentice Hall, New Jersey, 1993. 